\title{
Reporte de un caso clínico de latrodectismo en Ecuador y revisión bibliográfica
}

\author{
Report of a clinical case of latrodectism in Ecuador and literature review \\ Carlos Xavier Haro Erazo. ${ }^{1}$ \& María José Ortiz Rojas. ${ }^{2}$ \\ Recibido: 13-09-2019 / Revisado: 12-10-2019 /Aceptado: 22-10-2019/ Publicado: 08-11-2019
}

\begin{abstract}
DOI: https://doi.org/10.33262/cienciadigital.v3i4.1.999
\end{abstract}

Report of a clinical case of latrodectism in Ecuador and its literature review.

Latrodectism is caused by the bite of a spider of the genus Latrodectus, commonly called black widows, wheat spider or red pony spider. The species L. mactans, L. thoracicus, L. hasselti, among others, belong to this genus, which is widely distributed worldwide (4)

The venom of the genus Latrodectus contains $\alpha$-latrotoxin, a neurotoxin that acts at the presynaptic level of the autonomic nervous system (increasing the release of acetylcholine.) Producing a neurotoxic syndrome, which is difficult to diagnose unless there is clinical suspicion and a history of, I find with the arthropod there the importance of knowing about this pathology. The diagnosis is clinical, the treatment is reported pain management and support in addition to the use of anti-latrodectism antidote, the prognosis is favorable: a lethality between 0 and $6 \%$ is reported in different series (Jelinek, 1997; Schenone, 2003). (4)

Keywords: Latrodectism, neurotoxin, acetylcholine, antidote

\section{Resumen}

Reporte de un caso clínico de latrodectismo en Ecuador y su revisión bibliográfica.

El latrodectismo es causado por la mordedura de una araña del género Latrodectus, comúnmente llamadas viudas negras, araña del trigo o araña de poto colorado. Las

\footnotetext{
${ }^{1}$ Universidad Central del Ecuador, carlox.chex87@ hotmail.com

${ }^{2}$ Universidad Nacional de Chimborazo, majosortiz57@homtail.com
} 
especies L. mactans, L. thoracicus, L. hasselti, entre otras, pertenecen a este género, el cual está ampliamente distribuido a nivel mundial. (4)

El veneno del género Latrodectus contiene $\alpha$-latrotoxina, una neurotoxina que actúa a nivel presináptico del sistema nervioso autónomo (aumentando la liberación de acetilcolina.) produciendo un síndrome neurotóxico, el mismo que es de difícil diagnostico sino hay la sospecha clínica y el antecedente del encuentro con el artrópodo de ahí la importancia de conocer acerca de esta patología. El diagnóstico es clínico el tratamiento se reporta manejo del dolor y de soporte además del uso de antídoto anti latrodectismo, el pronóstico es favorable: se reporta una letalidad entre 0 y $6 \%$ en distintas series (Jelinek, 1997; Schenone, 2003). (4)

Palabras clave: Latrodectismo, neurotoxina, acetilcolina, antídoto

\section{Introducción}

Las arañas son artrópodos venenosos de distribución global. La mordedura de la mayoría de las arañas no tiene trascendencia clínica, debido a la dificultad que éstas tienen de inyectar veneno a los seres humanos por el pequeño tamaño de sus quelíceros. A pesar de esto, existen cientos de casos reportados con envenenamiento secundario a mordedura de arañas, la mayoría de información disponible proviene de países desarrollados.

Latrodectus mactans (viuda negra), es una araña pequeña, el macho regularmente no sobrepasa los $10 \mathrm{~mm}$ y pesa como máximo $0.2 \mathrm{~g}$, mientras que la hembra mide entre 30 y $40 \mathrm{~mm}$ y pesa hasta $1 \mathrm{gr}$, posee cuatro pares de patas de color negro brillante y una mancha tipo reloj de arena de color rojo intenso en el abdomen, el cual es globoso, tiene ocho ojos y tres uñas tarsales. El color varía según la especie, aunque por lo general es negro (5)

En los Estados Unidos, la Asociación Americana de Control de Envenenamientos (American Association of Poison Control Centers) reportó 18.000 víctimas de mordeduras de arañas en un periodo de 4 años. ${ }^{16}$ Solamente 4.000 de estos sujetos fueron admitidos a centros de salud, 54 pacientes tuvieron un mal pronóstico, pero ninguno murió a consecuencia del envenenamiento. En un estudio epidemiológico realizado en Australia, se confirmaron 750 casos de mordedura de arañas venenosas en un periodo de 27 meses. La escasa información proveniente de países en vías de desarrollo sugiere que, en regiones como América del Sur, las mordeduras de arañas 
son incluso más comunes que las de serpientes venenosas. En áreas rurales de Brasil, se reportaron aproximadamente 2,000 eventos de envenenamiento por animales durante 12 años, $59 \%$ de los que correspondieron a mordeduras de arañas y solo 17\% a serpientes. Sin embargo, la mayoría de casos de mordeduras de arañas fueron clínicamente leves, a diferencia de las mordeduras de serpientes, que cursan con morbimortalidad elevada.

\section{Caso Clínico}

Presentamos el caso de un Paciente masculino de 60 años de edad, sin antecedentes de importancia, residente en la parroquia Licto perteneciente a la provincia de Chimborazo, acude al servicio de urgencias, por haber sufrido picadura de araña a nivel de segundo dedo de mano derecha, 5 horas antes de su ingreso.

Refiere haber sufrido incidente mientras recogía piedras cerca de riachuelo, posterior a lo cual presenta intenso dolor en extremidad superior derecha que posteriormente progresaría hacia tórax, abdomen y extremidades inferiores.

Describe haber sufrido ataque por araña pequeña, de color negro, con tinte rojizo en el abdomen, características que coinciden con el género Latrodectus mactans (viuda negra), se comunica inmediatamente el suceso al Centro de Información y Asesoramiento Toxicológico (CIATOX) ubicado en la ciudad de Quito, para adquisición de antídoto.

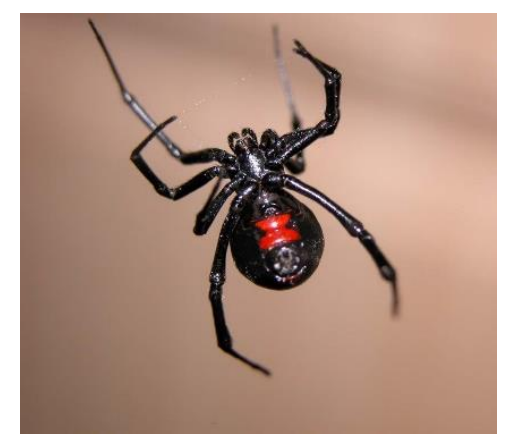

Figura 1. Viuda Negra

Fuente: https://hablemosdeinsectos.com/wp-content/uploads/2017/10/araña-viuda-negra1.jpg Al momento de su ingreso al área de observación paciente se encuentra irritable, álgico, febril, diaforético

- Tensión arterial 140/90

- Frecuencia cardiaca 107 por minuto

- Frecuencia respiratoria 20 por minuto

- Saturación de oxígeno $93 \%$ al aire ambiente 
- Temperatura $38^{\circ}$ centígrados

Como manejo inicial se establece hidratación a base de cristaliodes, esquema antibiótico con 1 gr de cefazolina, vacunación antitetánica, hidrocortisona 500 mg por vía intravenosa y analgesia a base de paracetamol y tramadol, al no conseguir disminución de dolor se instaura fentanyl en bomba de infusión, con lo que se logra control momentáneo de dolor.

\section{$\underline{\text { Exámenes complementarios al momento del ingreso }}$}

\begin{tabular}{|l|l|}
\hline Biometría hemática & $\begin{array}{l}\text { LEUCOCITOS 7.76, NEUTROFILOS 50 } \\
\%, \text { HB 19, HCTO 58 \%, PLAQUETAS } \\
246000\end{array}$ \\
\hline Gasometría arterial & $\begin{array}{l}\text { PH 7.40, PCO2 22.6, HCO3 13.8, EB -8.6, } \\
\text { PO2 62, SAT 90\% }\end{array}$ \\
\hline Coagulograma & TP 12.8, TTP 29.4, INR 1.06 \\
\hline Perfil Cardiaco & $\begin{array}{l}\text { TROPONINA 11.75, CPK 122, CKMB 99 } \\
\text { MIOGLOBINA 44 }\end{array}$ \\
\hline Química sanguínea & $\begin{array}{l}\text { GLUCOSA 148, UREA 43, BUN 20, } \\
\text { CREA 0.7, BT 0.36, AST 37, ALT 36, FA } \\
\text { 93, AMILASA 62 LIPASA 28 }\end{array}$ \\
\hline Electrolitos & CL 114, K 3.90, Na 143.0 \\
\hline
\end{tabular}

Tabla 1. Exámenes complementarios de ingresos

Fuente: Hospital General Riobamba, sistema AS400

Horas posteriores paciente con intenso dolor generalizado, taquicardia, hipertensión, taquipnea, alza térmica, desaturación y contractura muscular de pared abdominal.

- Tensión arterial 170/100

- Frecuencia cardiaca 117 por minuto

- Frecuencia respiratoria 26 por minuto

- Saturación de oxígeno $88 \%$ al apoyo de oxígeno por cánula nasal

- Temperatura $37,6^{\circ}$ centígrados

Se traslada paciente al área critica de urgencias, donde es valorado por terapia intensiva, ante el potencial riesgo de descompensación hemodinámica se decide su ingreso a cuidados intensivos. 
Exámenes complementarios en cuidados intensivos

\begin{tabular}{|l|l|}
\hline \multirow{2}{*}{ Biometría hemática } & $\begin{array}{l}\text { LEUCOCITOSIS 11.76, NEUTROFILIA } \\
92 \%, \text { HB 18, HCTO 52\%, PLAQUETAS } \\
234000\end{array}$ \\
\hline Gasometría arterial & $\begin{array}{l}\text { ACIDOSIS METABÓLICA NO } \\
\text { COMPENSADA }\end{array}$ \\
\hline Coagulograma & SIN CAMBIOS \\
\hline Perfil Cardiaco & $\begin{array}{l}\text { TROPONINA 11.75, CPK 122, CKMB 99 } \\
\text { MIOGLOBINA 44 }\end{array}$ \\
\hline Química sanguínea & $\begin{array}{l}\text { GLUCOSA 197, CREA 1.0. } \\
\text { RESTO SIN CAMBIOS }\end{array}$ \\
\hline EKG & $\begin{array}{l}\text { RITMO SINUSAL, BLOQUEO AV DE } \\
\text { PRIMER GRADO }\end{array}$ \\
\hline
\end{tabular}

Tabla 2. Exámenes complementarios cuidados intensivos

Fuente: Hospital General Riobamba, sistema AS400

En este momento paciente en condiciones delicadas, inestabilidad hemodinámica, fallo renal AKIN 1, hiperreflexia de extremidades.

- Tensión arterial 180/113

- Frecuencia cardiaca 109 por minuto

- Frecuencia respiratoria 25 por minuto

- Saturación de oxígeno $88 \%$ al apoyo de oxígeno por cánula nasal

- Temperatura $38^{\circ}$ centígrados

Se maneja hipertensión arterial con enalapirlato 1,25 intravenoso, control de hiperglicemia con esquema de insulina rápida por escapes, se mantiene pauta analgésica y esquema antibiótico. Horas posteriores a su ingreso a cuidados intensivos, se recibe antídoto, mismo que se administra inmediatamente 2 dosis por vía intravenosa, después de 1 hora presenta mejoría significativa de cuadro clínico.

- Tensión arterial 100/75

- Frecuencia cardiaca 73 por minuto

- Frecuencia respiratoria 18 por minuto

- Saturación de oxígeno 92 \% al apoyo de oxígeno por cánula nasal 
- $\quad$ Temperatura $37^{\circ}$ centígrados

Permanece tres días en área de cuidados intensivos, presenta diminución importante de dolor y contractura muscular, tolera vía oral, diuresis horaria adecuada.

Se traslada paciente hemodinamicamente estable al servicio de medicina interna, donde permanece bajo vigilancia estricta.

Se realiza control de laboratorio donde se reporta.

\begin{tabular}{|l|l|}
\hline \multirow{2}{*}{ Biometría hemática } & $\begin{array}{l}\text { LEUCOCITOS 4,00, NEUTROFILIA 80 } \\
\%, \text { HB 16, HCTO 51 \%, PLAQUETAS } \\
236000\end{array}$ \\
\hline Gasometría arterial & $\begin{array}{l}\text { PH 7.39, PCO2 34.4, HCO3 20,6, EB -3.4, } \\
\text { PO2 59,5, SAT 90\% }\end{array}$ \\
\hline Coagulograma & TP 12.1, TTP 30, INR 1.01 \\
\hline Perfil Cardiaco & $\begin{array}{l}\text { TROPONINA 21, CPK 149, CKMB 30.8, } \\
\text { MIOGLOBINA 175 }\end{array}$ \\
\hline Química sanguínea & $\begin{array}{l}\text { GLUCOSA 98, UREA 28, CREA 0.8, BT } \\
0.55, \text { AST 24, ALT 31, FA 93, AMILASA } \\
62 \text { LIPASA 31 }\end{array}$ \\
\hline Electrolitos & CL 105, K 3.90, Na 139.0 \\
\hline
\end{tabular}

Tabla 3. Control de Laboratorio

Fuente: Hospital General Riobamba, sistema AS400

Después de 6 días en total de hospitalización y al conseguir remisión completa de cuadro, paciente egresa en condiciones estables y con controles posteriores por consulta externa.

\section{Aspectos Clínicos:}

El cuadro clínico es pleomórfico, aunque pueden distinguirse tres síndromes principales: latrodectismo, loxoscelismo y envenenamiento por la araña de "tela en embudo". Latrodectus es el género que incluye las viudas negras y se distribuye en las regiones tropicales y subtropicales del planeta $(4,9)$

Puede mostrar dos tipos de manifestaciones: Locales es habitual la presencia de dolor punzante en el sitio de la picadura, que aumenta de intensidad y puede irradiarse o generalizarse y tornarse muy intenso. También pueden observarse una pápula eritematosa, leve edema, y piloerección, acompañados de hiperestesia local y contracturas musculares localizadas en el 
área de la picadura con tendencia a la generalización. (1)

Generales el cuadro sistémico se caracteriza por un síndrome de alarma que se traduce por opresión precordial, alteraciones del ECG, con taquicardia inicial e hipertensión seguidas de bradicardia, vómitos, hiperestesia generalizada, sudoración, sialorrea, midriasis con leve edema palpebral bilateral, hipersecreción nasal, bronquial y piloerección. Toda la sintomatología mencionada es de aparición rápida después de la picadura y evoluciona en forma de crisis, frecuentemente se presenta un cuadro de excitación y ansiedad pseudopsicótico (siempre con lucidez), con contracturas musculares dolorosas generalizadas y periódicas que, localizadas en abdomen, simulan un abdomen agudo. Suele asociarse paresia vesical e intestinal y en menor frecuencia priapismo, eyaculación, enuresis y oliguria. El óbito se produce en general por falla hemodinámica y edema agudo de pulmón, en algunos casos se presenta trismus, lo que, junto con la rubicundez facial, sudoración y blefaritis, constituyen la llamada "facies latrodectísmica". (1)

\section{Diagnostico}

El diagnóstico se basa en el antecedente de la picadura (de manera excepcional puede faltar este dato) asociado al cuadro clínico compatible. Si bien no existen exámenes complementarios específicos que faciliten el diagnóstico se recomienda solicitar: Hemograma. Glucemia. Uremia. Ionograma. Sedimento urinario. Amilasemia. CPK. ECG. $(1,5)$

El envenenamiento por Latrodectus puede ser fácilmente confundido con otros cuadros clínicos, en especial si no se ha visto el ejemplar agresor. Hay que establecer un diagnostico diferencial con: abdomen agudo infarto agudo de miocardio cólico renal torsión testicular intoxicación por inhibidores de la colinesterasa, en el embarazo puede simular una preclampsia (dolor abdominal e hipertensión) (6)

\section{Tratamiento}

El manejo es esencialmente sintomático y de soporte, siendo el pilar principal la analgesia. Se ha utilizado con éxito, como en este caso, la neostigmina en dosis 0,5-1 mg cada $8 \mathrm{~h}$ endovenoso, siendo suficiente una dosis en muchos casos (Schenone, 2003). Su uso se justifica por su inhibición reversible de la acetilcolinesterasa, que evitaría la depleción de acetilcolina y 
así los síntomas neuromusculares (Jelinek, 1997). Además, se ha propuesto el uso de gluconato de calcio $10 \%$, pero cada vez con menor uso en la práctica clínica, y benzodiacepinas (6) Específico ante el cuadro clínico evidente del envenenamiento, es importante la rápida instauración del tratamiento específico, que es la aplicación del antiveneno correspondiente. (1)

El CIATOX (Centro de Información y Asesoramiento Toxicológico) en el Ecuador es un departamento del Ministerio de Salud Pública al cual se debe notificar y reportar los casos clínicos para el correspondiente antídoto el mismo que está compuesto por fragmentos $\mathrm{F}$ (ab') de inmunoglobulinas y la presentación es en frasco ampolla de $2 \mathrm{ml}$.

\section{Discusión}

La picadura de araña, específicamente en este caso de latrodectus, aunque no sucede con regularidad en nuestra región, constituye un problema grave de salud, para quien lo padece.

Por lo general, el contacto con humanos se da en ambientes rurales, estos artrópodos se ubican en lugares soleados cerca del nivel del suelo ya sean hojas, rocas, base de arbustos y en plantaciones agrícolas.

La sintomatología no es específica, sin embargo, predominan las manifestaciones de tipo neurológico.

Cabe recalcar la importancia de la descripción de las características que pueda recordar la victima de la picadura, sobre el arácnido, con el fin de la orientación sobre la especie vinculada al ataque y garantizar el manejo clínico adecuado del paciente, tomando como puntos de importancia, la administración de antídotos.

La administración del antídoto en este caso y el manejo clínico oportuno, resultan clave para la recuperación satisfactoria del paciente.

Conocer las características de los arácnidos a lo que pueden estar expuestos para la pronta identificación y manejo médico.

En definitiva, este caso nos recuerda la importancia de conocer el manejo de patologías asociadas a ciertas actividades que se vinculen a la zona rural.

El primer mecanismo de acción de la a-latrotoxina sobre la membrana citoplasmática provoca la aparición de poros que llevan a la salida de cationes divalentes y monovalentes, como el $\mathrm{Ca}++, \mathrm{Mg}++, \mathrm{K}+\mathrm{y} \mathrm{Na}+$, de la célula, con la participación de dos moléculas de calcio; las toxinas pasan al interior de la célula por endocitosis y liberan gran cantidad de neurotransmisores como norepinefrina y acetilcolina. El segundo mecanismo está relacionado 
con la latrofilina, una proteína que sirve como receptor de la a-latrotoxina independiente de calcio, y se produce la liberación de ácido g-aminobutírico, que no depende de la acción del calcio. Como resultado de estos eventos ocurre la despolarización de la unión neuromuscular con hiperreactividad autonómica y agotamiento de la acetilcolina. El tercer mecanismo es mediado por la neurexina, el primer receptor descubierto para la a-latrotoxina, aunque el mecanismo exacto de acción por el cual la a-latrotoxina genera la salida de iones no se conoce completamente (5)

\section{Conclusiones}

1. El Latrodectismo en nuestro país es un cuadro clínico agudo con pocos datos epidemiológicos y desconocimiento sobre los signos y síntomas basados en el antecedente geográfico y situaciones puntuales que nos sirven de guía para tomar siempre en cuenta y no pasar por alto el latrodectismo.

2. El manejo del cuadro clínico puede inicialmente manejarse con analgesia y soporte de constantes vitales sin embargo no hay estudios suficientes que determinen que sea el manejo adecuado por lo cual la necesidad de un antídoto debe ser dado basado en el criterio del médico la evolución y condiciones del paciente.

3. Es sustancialmente importante las características de la araña para identificar el tipo de toxina a la que estamos enfrentando, sin embargo, la literatura nos permite tener un panorama más claro en base a la sintomatología y características clínicas de la mordedura.

4. Es importante el reporte epidemiológico en nuestro país con el objetivo de tener datos estadísticos que nos permitan estar más al tanto de la prevalencia e incidencia de casos de mordeduras de arañas en nuestro país.

\section{Referencias Bibliográficas}

1. Guía de prevención diagnóstico tratamiento y vigilancia epidemiológica de los envenenamientos por araña. Programa Nacional de Prevención y control de las intoxicaciones - PRECOTOX. Edición 2016 Ministerio de la Salud Presidencia de la Nación.

2. ARTICULO DE Revisión Arañas

Neurological Complications of Spider Bites Victor J. Del Brutto, MD, Oscar H. Del 
Brutto, MD Vol. 26, No 1, 2017 / Revista Ecuatoriana de Neurología.

3. Aspectos clínicos y epidemiológicosisếp de las mordeduras de araña en México Iván Renato Zúñiga-Carrasco y Janett Caro-Lozano Hosp Med Clin Manag. 2018; 11:191203 Articulo de Revisión

4. Latrodectismo. Caso clínico y revisión de la literatura Óscar Corsi S, Esteban Del Río O, Arturo Peña, David Acuña R. ARS MEDICA Revista de Ciencias Médicas Volumen

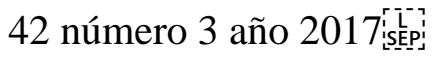

5. Latrodectismo en la provincia de Jujuy. Aspectos epidemiológicos y clínicos Latrodectism in the province of Jujuy. Epidemiological and clinical aspects Ojeda, Marta A., Remondegui, Carlos, Neder de Román, Lilia E. Acta Toxicol. Argent. (2015) 23 (3): $118-124$

6. Guía de referencia rápida diagnóstico y tratamiento de mordedura por arañas venenosas Gobierno Federal México Consejo de Salubridad General

7. Lactrodectismo en Costa Rica (Latrodectism in Costa Rica) Gilbert Barrantes-Montero y Raquel Valerio-Pizarro Acta méd costarric Vol 59 (2), abril-junio 2017

8. Comportamiento Epidemiológico de la Intoxicación por veneno de arañas en el Estado de México Inteligencia epidemiológica 2016 año 6 Núm. 1 enero Junio ISSN: 20075162 pp. $30-40$

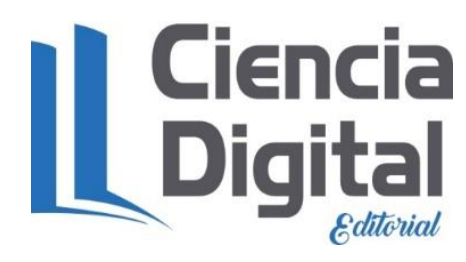




\section{PARA CITAR EL ARTÍCULO INDEXADO.}

Haro Erazo, C., \& Ortiz Rojas, M. (2019). Reporte de un caso clínico de latrodectismo en Ecuador y revisión bibliográfica. Ciencia Digital, 3(4.1), 126-136. https://doi.org/10.33262/cienciadigital.v3i4.1.999

\section{Ciencia \\ Ligital}

El artículo que se publica es de exclusiva responsabilidad de los autores y no necesariamente reflejan el pensamiento de la Revista Ciencia Digital.

El artículo queda en propiedad de la revista y, por tanto, su publicación parcial y/o total en otro medio tiene que ser autorizado por el director de la Revista Ciencia Digital.
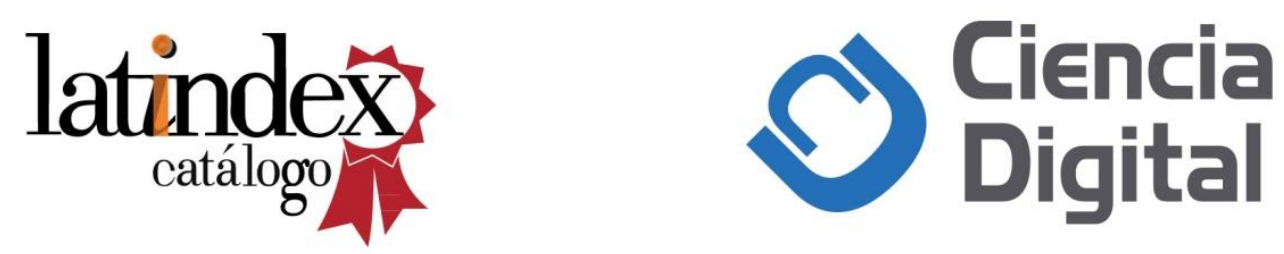